\title{
Electrospun oral formulations for combined photo-chemotherapy of colon cancer
}

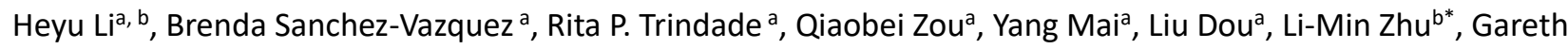

R. Williams ${ }^{\mathrm{a} *}$

a. UCL School of Pharmacy, University College London, 29-39 Brunswick Square, London, WC1N 1AX, UK

b. College of Chemistry, Chemical Engineering and Biotechnology, Donghua University, Shanghai, 201620, China

* Corresponding authors: Li-Min Zhu (Tel.: +86 21 67792659; email: Izhu@dhu.edu.cn ) and Gareth R. Williams (Tel.: +44 (0) 207753 5868; Email: g.williams@ucl.ac.uk)

10

Word count: 6286. 6 Figures, 2 tables.

\section{Abstract}

In this work, we report new formulations for the combined photo-chemotherapy of colon cancer. Fibers were fabricated via coaxial-electrospinning with the intent of targeting delivery of the anti-cancer drug carmofur (CAR) and the photosensitizer rose bengal (RB) selectively to the colon site. The fibers comprised a hydroxypropyl methylcellulose (HPMC) core loaded with the active ingredients, and a pH-sensitive Eudragit L100-55 shell. The fibers were found to be homogeneous and cylindrical and have visible core-shell structures. X-ray diffraction and differential scanning calorimetry demonstrated that both CAR and RB were present in the fibers in the amorphous physical form. In vitro drug release studies showed that the fibers have the potential to selectively deliver drugs to the colon, with only $10-15 \%$ release noted in the acidic conditions of the stomach but sustained release at $\mathrm{pH}$ 7.4. Cytotoxicity studies were undertaken on human dermal fibroblast (HDF) and colon cancer (Caco-2) cells, and the influence of light on cell death was also explored. The fibers loaded with CAR alone showed obvious toxicity to both cell lines, with and without the application of light. The RB-loaded fibers led to high viability (ca. $80 \%$ for both cell types) in the absence of light, but much greater toxicity was noted $(30-60 \%)$ with light. The same trends were observed with the formulation containing both $C A R$ and $R B$, but with lower viabilities. The RB and RB/CAR loaded systems show clear selectivity for cancerous over non-cancerous cells. Finally, mucoadhesion studies revealed there were strong adhesive forces between the rat colonic mucosa and the fibers after they had passed through an acidic environment. Such electrospun fibers thus could have potential in the development of oral therapies for colon 
cancer.

Keywords: electrospinning, colon cancer, oral administration, drug delivery, mucoadhesion, photochemotherapy

\section{Introduction}

Colon cancer is a major cause of death globally [1]. Treatment is based largely on the stage (extent) of the cancer. In most cases, surgery will be conducted to remove the affected section of the colon, along with nearby lymph nodes. Chemotherapy is typically applied in addition, before and/or after surgery, as an adjuvant therapy. For advanced cancers, radiation therapy can also be used [2-4]. Unfortunately, chemotherapy usually results in unpleasant side effects owing to its non-specificity: it not only kills cancer cells, but also damages healthy tissues. Therefore, photodynamic therapy (PDT) has attracted increasing attention as an alternative adjuvant therapy.

For PDT, a photosensitizer is applied. This generates cytotoxic reactive oxygen species (ROS) upon excitation with visible or near infrared light. Because of the requirement for activation by light, PDT can be used to provide treatment locally at the irradiated areas. The ROS produced in PDT do not affect the collagen or elastin of healthy tissue, and hence PDT can result in local death of cancer cells without side effects [5]. Further, the combination of PDT with chemotherapy in a single formulation offers a potential route to potent but selective treatment of cancer [6-8].

Electrospinning is a method which can be used to fabricate non-woven one-dimensional fibers with diameters on the micro- or nanoscale $[9,10]$. Fibers produced by electrospinning have attracted increasing attention in the field of drug delivery because they have several intrinsic benefits, including high porosity, tunability, a high surface area-to-volume ratio, and a similar structure to the extracellular matrix in tissues [10]. Further, the resultant fibers can have high ( $>50 \% \mathrm{w} / \mathrm{w}$ ) drug loadings and good biocompatibility, essential properties for drug delivery systems (DDSs) [11-16].

A number of researchers have explored electrospun DDSs for cancer treatment and obtained promising results. For example, Chen et al. constructed a dual-drug carrier system [17]. Core-shell nanoparticles were 
first loaded with the anticancer drug doxorubicin before being incorporated into fibers comprising poly $(\varepsilon$ caprolactone) and gelatin co-loaded with the antiphlogistic drug indomethacin. The resultant multifunctional fibers could be surgically implanted directly at the tumor site in mice and used for simultaneous chemotherapy and imaging. In other work, Kaplan et al. applied a local drug delivery strategy to combat lung cancer recurrence after tumor resection using a biodegradable electrospun nanofiber mesh loaded with cisplatin [18]. Fibers were fabricated from poly( $\varepsilon$-caprolactone) and poly(glycerol monostearate-cocaprolactone) to afford favorable mechanical properties. They showed extended release of cisplatin in a linear fashion over 90 days, with anti-cancer efficacy demonstrated using an in vitro Lewis lung carcinoma cell assay. An in vivo evaluation resulted in a significant increase in the median recurrence-free survival time. These recent results emphasize the potential of electrospun fibers for improving prognosis for lung cancer patients undergoing tumor resection.

The body of work in the literature to date thus suggests that electrospun nanofibers have considerable potential for use in cancer treatment [19]. However, no studies to date have probed the combination of PDT with chemotherapy via electrospun formulations. In this work, we incorporated a model anti-cancer drug (carmofur; CAR) and photosensitizer (rose bengal; RB) into the core of core-shell fibers via coaxial electrospinning. Hydroxypropyl methylcellulose (HPMC) was used as the core polymer, because it is well known to be mucoadhesive [20]. Mucoadhesive drug delivery systems are advantageous since the entire DDS will be rendered immobile in the desired physiological environment, as a result of intimate contact between 80 the DDS and mucosa. This results in a high drug concentration at the absorption surface, and thereby a reduction in the required dose together with improved bioavailability [21-23]. For the shell, we used Eudragit L100-55 (methacrylic acid - ethyl acrylate copolymer type A, 1:1 molar ratio), which is highly soluble in water above $\mathrm{pH} 5.5$ but insoluble below this $\mathrm{pH}$. A series of formulations were produced by coaxial electrospinning. After oral administration, the Eudragit shell should prevent the release of CAR and RB as the formulation passes through the stomach. Later, the shell will dissolve to expose the HPMC core to the intestinal fluids and the latter will adsorb water, swell, and adhere to the colon walls. Detailed morphological and physicochemical characterization, in vitro drug release, cell viability, and mucoadhesion assays are reported. 


\section{Experimental}

\section{Materials}

Carmofur (CAR) was purchased from Santa Cruz Biotechnology (UK), while rose bengal disodium salt (RB, 95\%), anhydrous ethanol, 1,1,1,3,3,3-hexafluoro-2-propanol (HFIP, $\geq 99.5 \%)$ and hydrochloric acid were provided by Sigma-Aldrich (UK). Benecel ${ }^{\mathrm{TM}}$ hydroxypropylmethylcellulose K4M (HPMC; Mw 400,000 Da) was supplied by Ashland Inc. (Switzerland). Eudragit L100-55 (Mw ca. 135,000 Da) was sourced from Evonik GmbH (Germany). Phosphate buffered saline (PBS, pH7.4) tablets were supplied by VWR Life Science (UK). The Caco2 colorectal adenocarcinoma cell line was obtained from ATCC (UK). Human dermal fibroblast (HDF) cells, Dulbecco's modified Eagle's medium high-glucose (DMEM-HG), penicillin-streptomycin, L-glutamine, nonessential amino acid solution and heat-inactivated fetal bovine serum were supplied by Life Technologies (UK). All water was deionized before use.

\section{Preparation of electrospinning solutions}

For the shell solution, Eudragit L100-55 was dissolved in ethanol at $10 \% \mathrm{w} / \mathrm{v}$, with magnetic stirring performed overnight to ensure complete dissolution. Solutions of HPMC were prepared in HFIP at $1 \% \mathrm{w} / \mathrm{v}$. CAR and RB were added into the HPMC solution at a concentration of $60 \%$ and $15 \% \mathrm{w} / \mathrm{w}$ with respect to the mass of HPMC. A solution of HPMC alone and HPMC solutions containing only $60 \% \mathrm{w} / \mathrm{w}$ CAR or $15 \% \mathrm{w} / \mathrm{w}$ RB were prepared as controls. Full details of the solutions are presented in Table 1.

Table 1. Details of the spinning solutions used in this work.

\begin{tabular}{ccccc}
\hline \multirow{2}{*}{ Sample } & \multicolumn{2}{c}{ Core solution contents $(\% \mathbf{w} / \mathbf{w})^{\text {a }}$} & \multicolumn{2}{c}{ Fiber contents $(\% \mathbf{w} / \mathbf{w})$} \\
\cline { 2 - 5 } & CAR & RB & CAR & RB \\
\hline S1 & ---- & --- & --- & -- \\
S2 & 60 & ---- & 1.9 & -- \\
S3 & ---- & 15 & --- & 0.5 \\
S4 & 60 & 15 & 1.9 & 0.5 \\
\hline
\end{tabular}

110 a With respect to the mass of HPMC

\section{Electrospinning}

The core and shell solutions were separately loaded into $5 \mathrm{~mL}$ plastic syringes and attached to a coaxial spinneret (inner needle internal diameter (ID) $0.34 \mathrm{~mm}$; outer needle ID $1.12 \mathrm{~mm}$ ). The syringes were mounted separately on two independent syringe pumps (KDS100, KD Scientific, USA) and the positive electrode of a high voltage power supply (HCP35-35,000, FuG Elektronik, Germany) was attached to the outer 
needle. During the spinning process, the flow rates of the shell and core solutions were set at 0.6 and $0.2 \mathrm{~mL}$ $\mathrm{h}^{-1}$, respectively. A voltage of $20 \mathrm{kV}$ was applied between the spinneret and a grounded collector (a flat piece of aluminum foil of $10 \times 10 \mathrm{~cm}$ in size). The distance between the needle tip and the grounded collector was fixed at $18 \mathrm{~cm}$. The relative humidity was $c a .40 \%$, and the temperature approximately $25^{\circ} \mathrm{C}$. After electrospinning for $8 \mathrm{~h}$, the products were collected and stored in a desiccator at room temperature for $24 \mathrm{~h}$ to remove any residual solvent. A $1 \% \mathrm{w} / \mathrm{v}$ HPMC solution in HFIP was also processed with monoaxial electrospinning. In this case, a stainless-steel flat tipped needle (20G, 0.61 mm inner diameter, Nordson EFD) was employed as the spinneret. The flow rate was $0.6 \mathrm{~mL} \mathrm{~h}^{-1}$, and all other parameters were the same as for coaxial electrospinning.

\section{Morphology}

The fiber morphology was observed by scanning electron microscopy (SEM; Quanta 200F instrument, FEI, the Netherlands) at a voltage of $5 \mathrm{kV}$. Samples were cut from the fiber mats and sputter coated with gold for 60 $s$ under argon. The mean diameter of the fibers in each sample was quantified using the ImageJ software (National Institutes of Health, USA) to make measurements at more than 100 points in each of the SEM images. To probe the internal structure of the coaxial fibers, a CM120 Bio-Twin transmission electron microscope (TEM; Philips/FEI Corporation, the Netherlands) was employed. Samples were collected by fixing a lacey carbon-coated copper grid directly onto the collector during the electrospinning process, and depositing fibers on it for around $30 \mathrm{~s}$.

\section{Fiber characterization}

X-ray diffraction (XRD) data were acquired on a MiniFlex 600 diffractometer (Rigaku, Japan) supplied with $\mathrm{Cu}$ K $\alpha$ radiation $(\lambda=1.5418 \AA$ ) at a voltage of $40 \mathrm{kV}$ and current of $15 \mathrm{~mA}$. Patterns were collected over the $2 \theta$ range 3 to $35^{\circ}$ at a speed of $5^{\circ} \mathrm{min}^{-1}$. Fourier transform infrared (FTIR) spectroscopy was performed using a Spectrum 100 instrument (PerkinElmer, USA). Spectra were collected over the range 4000 to $650 \mathrm{~cm}^{-1}$, with the spectral resolution set at $1 \mathrm{~cm}^{-1} .4$ scans for each sample were recorded.

Differential scanning calorimetry (DSC) was undertaken with the aid of a Q2000 calorimeter (TA instruments, USA). Non-hermetically sealed samples in aluminium pans (Tzero premium pan/lid, TA instruments, USA) were heated from 10 to $300{ }^{\circ} \mathrm{C}$ at $10{ }^{\circ} \mathrm{C} \mathrm{min}^{-1}$ under a nitrogen flow of $50 \mathrm{~mL} \mathrm{~min}$. Data were analyzed using the TA Instruments Universal Analysis software. 


\section{In vitro drug release}

$30 \mathrm{mg}$ of fibers were added into a sinker and release studies performed at $37 \pm 0.5{ }^{\circ} \mathrm{C}$ under mechanical stirring at $50 \mathrm{rpm}$. Samples were first placed in $50 \mathrm{~mL}$ of a pH 1.2 hydrochloric acid solution for $2 \mathrm{~h}$, to mimic the gastric fluid. Subsequently, samples were transferred to $50 \mathrm{~mL}$ of $\mathrm{pH} 7.4$ phosphate buffered saline (PBS) for $24 \mathrm{~h}$, to imitate the intestinal fluid. At periodic intervals, $1 \mathrm{~mL}$ samples were removed, and $1 \mathrm{~mL}$ of preheated $\mathrm{HCl}$ or PBS added to ensure a constant volume was maintained. A calibration curve was constructed by UV spectroscopy (6305 spectrophotometer, Jenway, UK) and used to calculate the drug concentrations in the aliquots. The absorbance was measured at wavelength of $260 \mathrm{~nm}$ for CAR and $547 \mathrm{~nm}$ for RB. Dissolution tests were carried out three times, and the results are reported as mean \pm standard deviation.

\section{Cytotoxicity}

These studies were carried out using humancolorectal adenocarcinoma (Caco-2, ATCC (UK), HTB-37) and human dermal fibroblast cells (HDF, Life Technologies (UK), lot 771555). The cells were cultured in Dulbecco's modified Eagle's medium (DMEM) supplemented with $10 \% \mathrm{v} / \mathrm{v}$ heat-inactivated fetal bovine serum (FBS), $1 \%$ v/v penicillin-streptomycin, $1 \%$ v/v MEM non-essential amino acids and $1 \%$ v/v glutamine. Culture was performed at $37{ }^{\circ} \mathrm{C}$ in a $5 \% \mathrm{CO}_{2}$ atmosphere. The cells were passaged every 3 days and reseeded at a concentration of $10^{6}$ cells $\mathrm{mL}^{-1}$ prior to use.

Fibers were dissolved in complete DMEM at a concentration of $3 \mathrm{mg} \mathrm{mL}^{-1}$ (giving equivalent drug concentrations of $57 \mu \mathrm{g} \mathrm{mL}^{-1} \mathrm{CAR}$ and/or $15 \mu \mathrm{g} \mathrm{mL} \mathrm{L}^{-1} \mathrm{RB}$ ). These solutions were then filtered through a 0.22 $\mu \mathrm{m}$ filter. $180 \mu \mathrm{L}$ of each solution was added to 96-well plates (Greiner Bio-One Cellstar, Sigma Aldrich (UK)) with cells suspended in them. The final cell density was $5 \times 10^{4}$ cells $\mathrm{mL}^{-1}$. Pure CAR and RB dissolved in DMEM were used as controls. The effect of light on the cytotoxicity of the formulations was explored by irradiating the cells at $521 \mathrm{~nm}$ under a LED microscope illuminator (DiCon, USA) for $20 \mathrm{~min}$ (1050 mW, $0.32 \mathrm{~cm}^{2}$ illumination area) after they had been incubated with the formulations for $24 \mathrm{~h}$. Control experiments were also performed without the application of light.

The CellTiter-Glo ${ }^{\text {TM }}$ luminescent cell viability assay (Promega, USA) was applied to measure cell viability. The 
luminescent reagent was prepared following the manufacturer's instructions and added to the culture plates with a reagent volume equal to the volume of culture medium present in each well. The plates were then incubated at room temperature for 30 min before a SpectraMax M2e spectrophotometer (Molecular Devices, USA) was used to determine luminescence. Data are reported as mean \pm standard deviation from three independent experiments, with three replicates in each experiment. The formula used for calculating the cell viability is as follows:

$$
\% \text { viability }=\frac{\text { Fluorescence of sample }- \text { background }}{\text { Fluorescence of untreated cells control }- \text { background }} \times 100 \%
$$

\section{Mucoadhesion}

Mucoadhesion was assessed using a tensile strength method (Instron, UK) following protocols reported in the literature [24]. Frozen large intestine from Sprague-Dawley rats was defrosted slowly at $10^{\circ} \mathrm{C}$ overnight, to minimize damage to the mucosa. Subsequently samples were allowed to warm to room temperature, before being further warmed to $37^{\circ} \mathrm{C}$ in a water bath. The tissue was not subjected to prehydration.

Samples of the mucosa $\left(2 \mathrm{~cm}^{2}\right)$ were cut and placed on a petri dish. A cylindrical home-made HDPE (high density polyethylene) adaptor (height: $10 \mathrm{~mm}$; diameter: $10 \mathrm{~mm}$ ) was attached to the probe of the tensile tester using double-sided tape. The surface of the HDPE adaptor was then covered with a sample cut from a fiber mat, with the fibers secured to the adaptor using double-sided tape (see Supplementary Material. Figure S1). The fiber-covered adaptor was moved down toward the mucosa at a constant speed of $10 \mathrm{~mm} \mathrm{~min}^{-1}$, and a compressive force of $0.5 \mathrm{~N}$ applied for $5 \mathrm{~min}$. The adaptor was next moved away from the mucosa at a constant speed of $20 \mathrm{~mm} \mathrm{~min}^{-1}$. The mucoadhesive properties were evaluated in terms of the energy at break, defined as the work of adhesion [25]. Measurements were performed in triplicate, with experiments using the adaptor without fibers mounted employed as a negative control. Neat HPMC fibers prepared through single-fluid electrospinning were attached to the adaptor without any pre-treatment. The coaxial fibers first underwent a dissolution stage (2 $\mathrm{h}$ in $\mathrm{pH} 1.2 \mathrm{HCl}$, then 1, 2, or $4 \mathrm{~h}$ in a $\mathrm{pH} 7.4 \mathrm{PBS}$ buffer), and then were retrieved from the medium and dried at room temperature before measurement. 


\section{Results and discussion}

\section{Fiber morphology}

SEM images and the diameter distribution of the fibers are depicted in Figure 1. All the samples appear homogeneous and smooth, with regular cylindrical morphologies. The mean diameter of the drug-free nanofibers S1 was $226 \pm 73 \mathrm{~nm}$, similar to the RB-loaded fibers S3 $(238 \pm 72 \mathrm{~nm})$. However, the fibers loaded with CAR (S2) and both active ingredients (S4) showed slightly reduced mean diameters, at $200 \pm 72 \mathrm{~nm}$ and $196 \pm 57 \mathrm{~nm}$, respectively. In both S2 and S4, a sub-population of a small number of very narrow fibers can be seen.
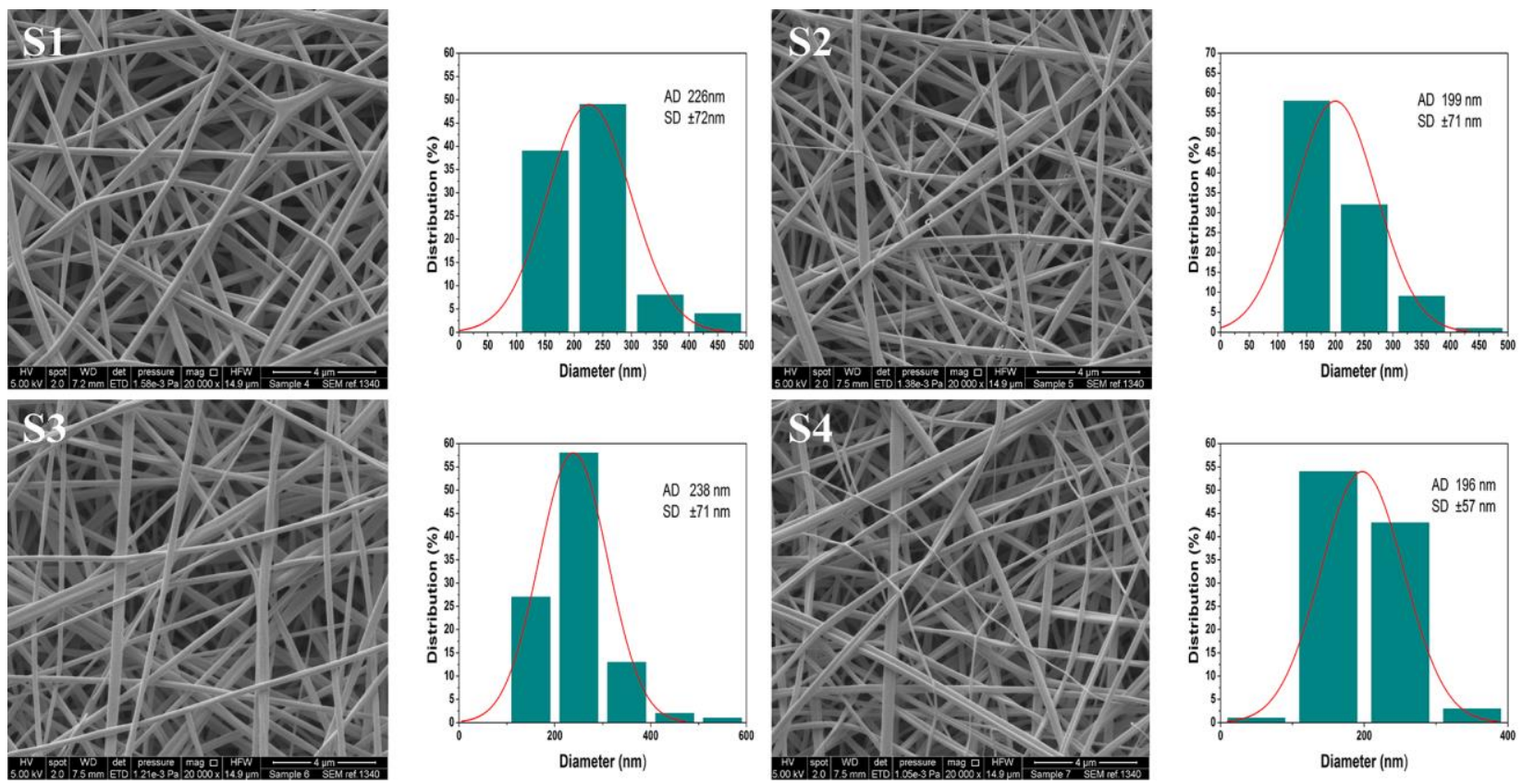

Figure 1. SEM images and the diameter distributions of the fibers (AD: mean diameter). Scale bar: $4 \mu \mathrm{m}$.

To investigate the internal structure of the fibers, TEM images were obtained (Figure 2). The core-shell structures of the fibers are clearly visible, confirming that coaxial spinning had been successfully performed. 

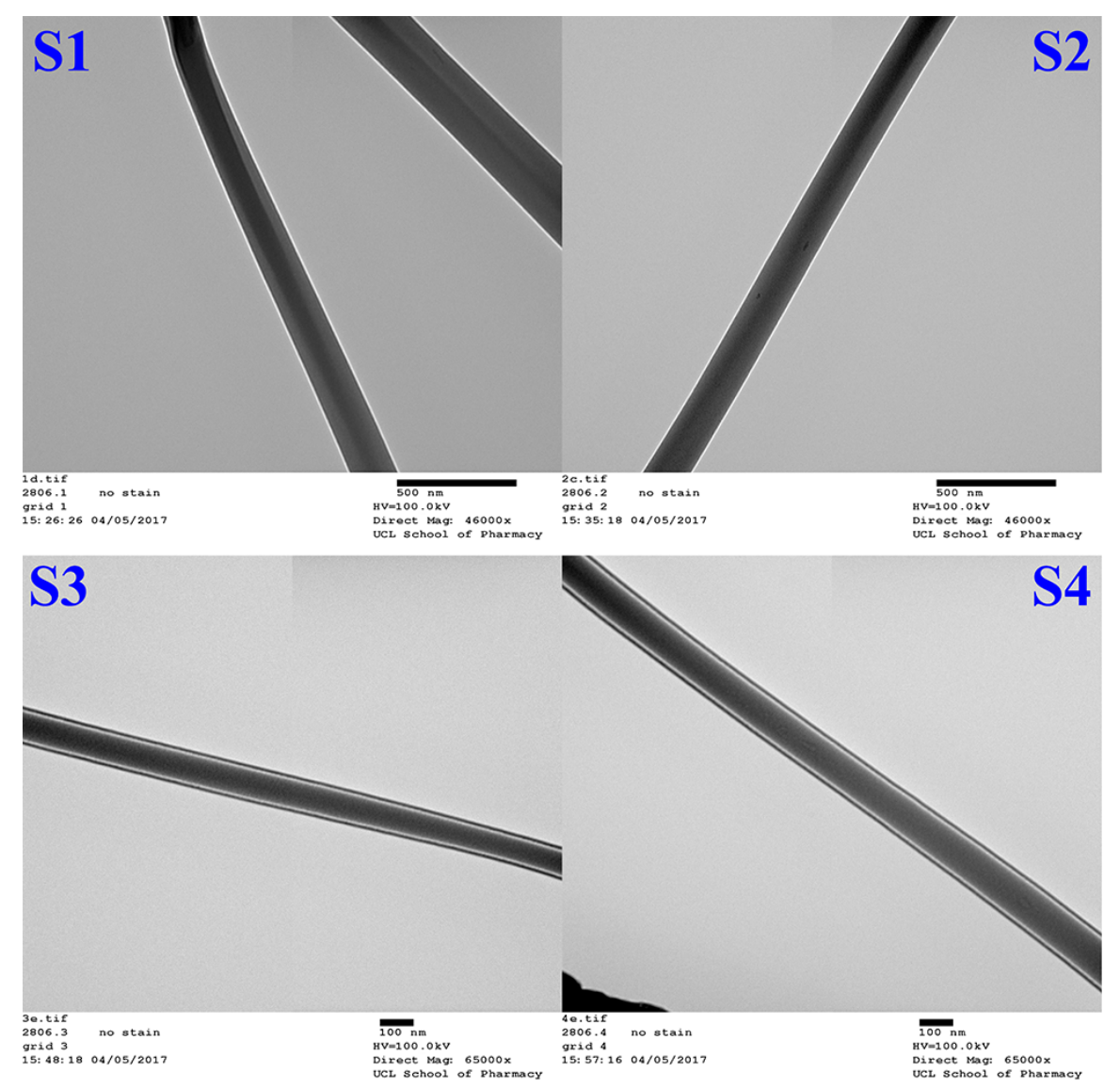

Figure 2. TEM images of the fibers. Scale bars: $500 \mathrm{~nm}(\mathrm{~S} 1, \mathrm{~S} 2)$ or $100 \mathrm{~nm}(\mathrm{~S} 3, \mathrm{~S} 4)$.

225

\section{X-ray diffraction}

XRD patterns of the fiber samples (S1-S4) and pure CAR and RB are given in Figure 3a. CAR and RB exhibit numerous sharp Bragg reflections, showing the raw materials to be crystalline in nature. The patterns of the four fiber samples do not show any distinct Bragg reflections, and all that can be seen are broad haloes. The distinct reflections of the pure drugs have disappeared in the patterns of the drug-loaded fibers (S2-S4), which suggests the formation of amorphous solid dispersions. 


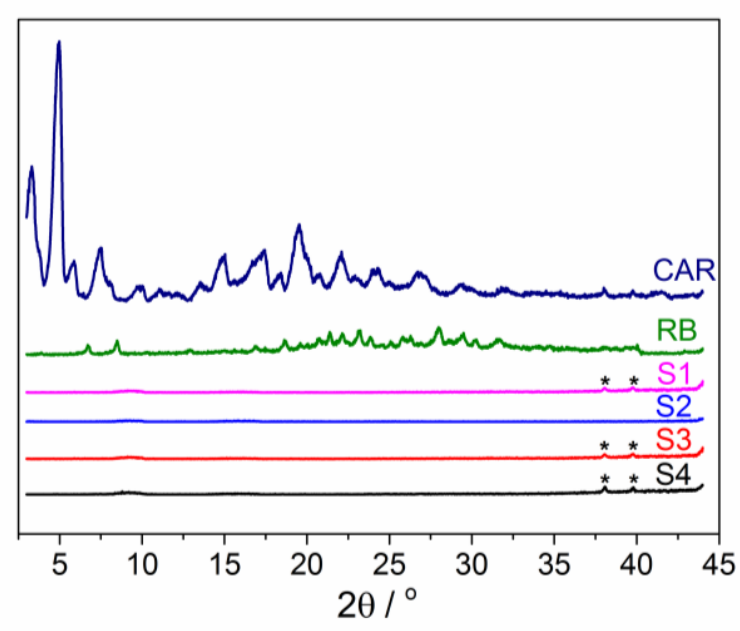

(a)

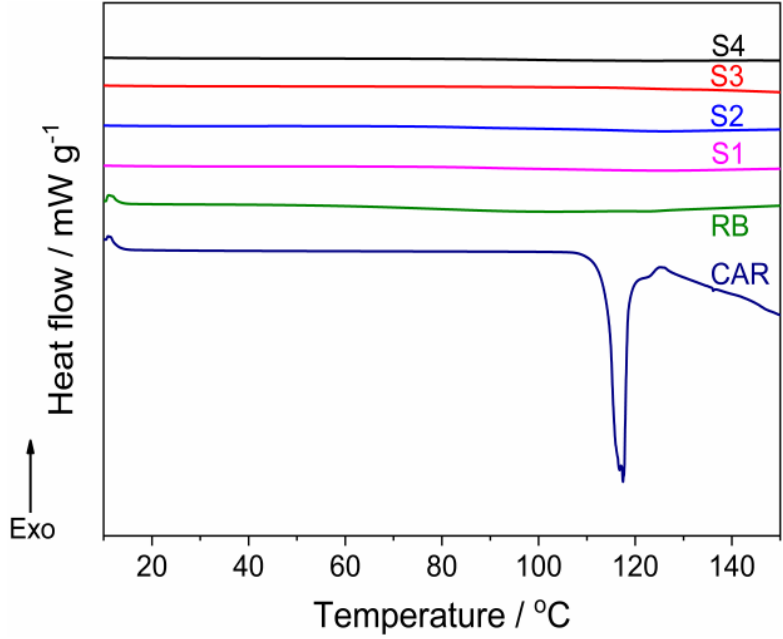

(b)

Figure 3. (a) XRD patterns and (b) DSC traces of CAR and RB and the electrospun fibers. Reflections marked * correspond to the sample holder.

\section{DSC}

DSC thermograms are presented in Figure 3b. CAR displays a distinct endothermic melting peak at around $115^{\circ} \mathrm{C}$, which agrees with the literature and verifies its crystalline nature [7]. This is followed by what appears to be degradation. RB shows no melting events over the temperature range explored (Figure $\mathbf{3 b}$ ), which is expected since its melting point is reported to be above $300{ }^{\circ} \mathrm{C}$ [7]. Because the other materials used in the formulations degrade significantly at the high temperatures needed to melt RB, it was not possible to run the DSC experiments to visualize this.

No fusion events are visible in the DSC thermograms for the fibers, consistent with the conversion of CAR into the amorphous state after electrospinning. These results agree with those from XRD, and similar conclusions have been reported many times in the literature for electrospun formulations [26-28]. It is widely recognized that the solvent evaporation process during electrospinning is very rapid, meaning there is insufficient time for the drug molecules to order themselves into a crystalline arrangement during drying. The random arrangement of molecules in the solution phase is carried through into the solid state, leading to a solid solution $[29,30]$.

The other key features that might be expected in the DSC data are $T_{\mathrm{g}} \mathrm{s}$ from the polymers. The $\mathrm{T}_{\mathrm{g}}$ of HPMC is reported to range from 162 to $170{ }^{\circ} \mathrm{C}$ [31], and that for Eudragit L100-55 is ca. $110{ }^{\circ} \mathrm{C}$. A close inspection of the DSC data (Figure S2) tentatively suggest that $\mathrm{T}_{\mathrm{g}}$ events may be visible at around $90-110^{\circ} \mathrm{C}$, but these are 
conflated with complex endothermic events believed to arise from the degradation of the CAR and/or polymer components of the formulations. The latter also overlap with the range over which the HPLC $T_{g}$ would be expected (given the plasticising influence of the active ingredients in the core, this would arise somewhere below $160^{\circ} \mathrm{C}$ ). Thus, the presence of $\mathrm{T}_{\mathrm{g}} \mathrm{s}$ cannot be unambiguously determined. Nevertheless, the DSC data are fully consistent with the XRD data, and confirm the formation of amorphous solid dispersions.

\section{FTIR spectroscopy}

FITR spectra of the fibers and the raw materials (RB, CAR, HPMC and Eudragit L100-55) are given in Figure 4a. Raw RB shows a peak at about $1615 \mathrm{~cm}^{-1}$, corresponding to its carbonyl stretch. The $\mathrm{C}=\mathrm{O}$ stretching vibrations of CAR appear from $1659-1717 \mathrm{~cm}^{-1}$ and at $1507 \mathrm{~cm}^{-1}$. For the raw polymer HPMC, vibrations arising from $\mathrm{OH}$ groups $\left(3424 \mathrm{~cm}^{-1}\right)$ and $\mathrm{C}-\mathrm{H}$ stretches $\left(2900 \mathrm{~cm}^{-1}\right)$ can be seen. A further characteristic peak at around 1060 $\mathrm{cm}^{-1}$ arises from C-O-C stretches. Eudragit L100-55 presents a peak at $1155 \mathrm{~cm}^{-1}$, which corresponds to ester stretching bands, and a strong absorption at $1731 \mathrm{~cm}^{-1}$ caused by the stretching of the carbonyl groups.

For all the fibers, the characteristic peaks from both HPMC (C-O-C and C-H stretches) and Eudragit L100-55 270 ( $\mathrm{C}-\mathrm{O}-\mathrm{C}$ and $\mathrm{C}=\mathrm{O}$ stretching) can be observed, indicating the successful incorporation of the two polymers in the fibers. Some peak shifts can also be seen. For instance, the Eudragit C-H shift moves from $2932 \mathrm{~cm}^{-1}$ in the pure polymer to $2928-2933 \mathrm{~cm}^{-1}$ in the fiber formulations, and its $C=0$ peak moves from $1731 \mathrm{~cm}^{-1}$ to $1729-1732 \mathrm{~cm}^{-1}$. These shifts indicate interactions between the polymers and/or drug molecules in the fibers. The presence of RB in the fibers cannot clearly be confirmed from the IR spectra. The major RB peaks all overlap with those of the polymer carriers, and the drug loading is also low at $0.5 \% \mathrm{w} / \mathrm{w}$. The presence of CAR is also difficult to discern, but compared with $\mathrm{S} 1$ and $\mathrm{S} 3$, the $\mathrm{C}=0$ peaks (at around $1730 \mathrm{~cm}^{-1}$ ) in the CARloaded fibers S2 and S4 appear to be enhanced in intensity, which might demonstrate the successful incorporation of CAR in these fibers. 


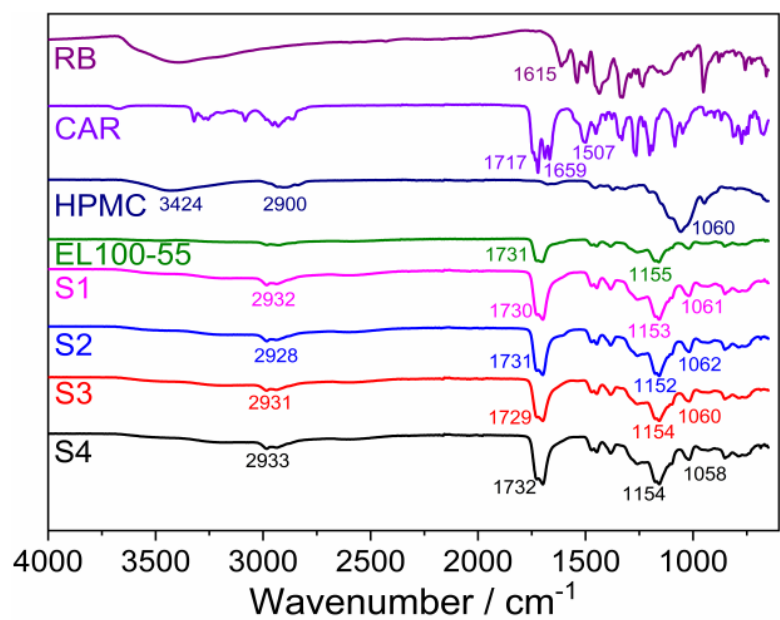

(a)

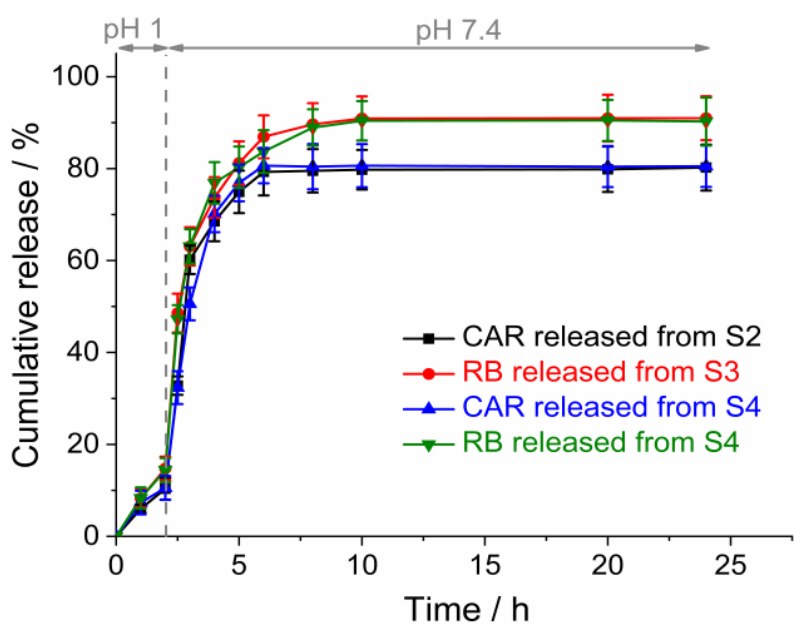

(b)

Figure 4. (a) FTIR spectra of the fibers and raw materials. (b) Drug release profiles from the S2, S3 and S4 fibers. Data are given as mean \pm S.D. from three independent experiments.

\section{Drug release}

To mimic the conditions of the human gastrointestinal tract, drug release experiments were first carried out in an acidic solution $(0.1 \mathrm{M} \mathrm{HCl})$ to stimulate the gastric fluid, and then in a PBS buffer $(\mathrm{pH} 7.4)$ to simulate intestinal fluid. The release profiles obtained are shown in Figure $\mathbf{4 b}$. In the first two hours, only $10.3 \%$ of the CAR loading was released from S2. After being transferred to the PBS buffer, sustained release was observed over the next $4 \mathrm{~h}$ until $80.3 \%$ release was attained. Similarly, for S3 only $14.8 \%$ of the RB content was released in $\mathrm{HCl}$, and this was followed by sustained release in PBS until $90.1 \%$ of the drug cargo was freed into solution at $10 \mathrm{~h}$. For the fibers loaded with both CAR and RB (S4), the release profiles of the two drugs were similar to those from S2 and S3.

These $\mathrm{pH}$-dependent release profiles can be attributed to the $\mathrm{pH}$-sensitive shell. Eudragit $\mathrm{L} 100-55$ is only soluble in aqueous conditions above $\mathrm{pH} 6.0$, when sufficient $\mathrm{COOH}$ groups in the polymer chain become ionized to permit it to dissolve. As a result, the Eudragit shell can minimize the release of both CAR and RB in acidic conditions, but triggers sustained release when in PBS. This release behavior is promising for the treatment of intestinal diseases via oral administration. The formulations will free only a small portion of their drug loading in the stomach. However, when the fibers reach the later parts of the intestine, which are at higher $\mathrm{pH}$, the shell will dissolve, releasing the drug cargo. The US Pharmacopoeia states that for delayed release dosage forms, less than $10 \%$ of the incorporated drug should be released in the acidic media, and the fibers perform in line with this requirement. Therefore, the core-shell fibers loaded with CAR and RB could 
comprise potent delayed-release oral treatments for colon cancer.

\section{Cytotoxicity}

305 The results of cytotoxicity studies performed on HDF and Caco-2 cells can be found in Figure 5 . The solutions of CAR all led to low cell viability (with and without light exposure), indicating its strong cytotoxicity to both cell lines. CAR is an antineoplastic pro-drug which can prevent DNA synthesis by cells, and thus causes reduced viability [32]. Moreover, the active degradation product from CAR is 5-fluorouracil, which has low selectivity to tumor cells and causes widespread damage to healthy tissues [33]. The viability of both types of cells cultured with RB solution was significantly lower with than without light exposure. This is because, as reported in the literature, light exposure is essential for the generation of cytotoxic reactive oxygen species by RB [34]. A combination of RB and light treatment showed some selectively for tumor cells. The viability of HDF cells treated with the RB solution and light was $51.4 \pm 13.1 \%$, higher than that of Caco-2 cells ( $26.5 \pm 7$. $8 \%)$.

The drug-free S1 fibers showed good viabilities (around 90\%) with both cell types, with and without light exposure. In contrast, the CAR-loaded S2 fibers killed around $50-60 \%$ of the cells in all cases, as a result of the potent cytotoxicity of the drug. The S3 fibers showed similar effects to an RB solution, with promising selectivity to tumor cells upon light exposure. For the S4 fibers loaded with both CAR and RB, the toxicity of CAR and selectivity of RB were combined. Only $16.5 \pm 6.6 \%$ of the Caco- 2 cells survived after being cultured with S4 fibers and exposed to light, the highest cytotoxicity observed in the experiment. This result can be attributed to the combination of photodynamic therapy and chemotherapy, which has been shown to lead to significant inhibition of tumor proliferation, and increased induction of apoptosis $[35,36]$. 


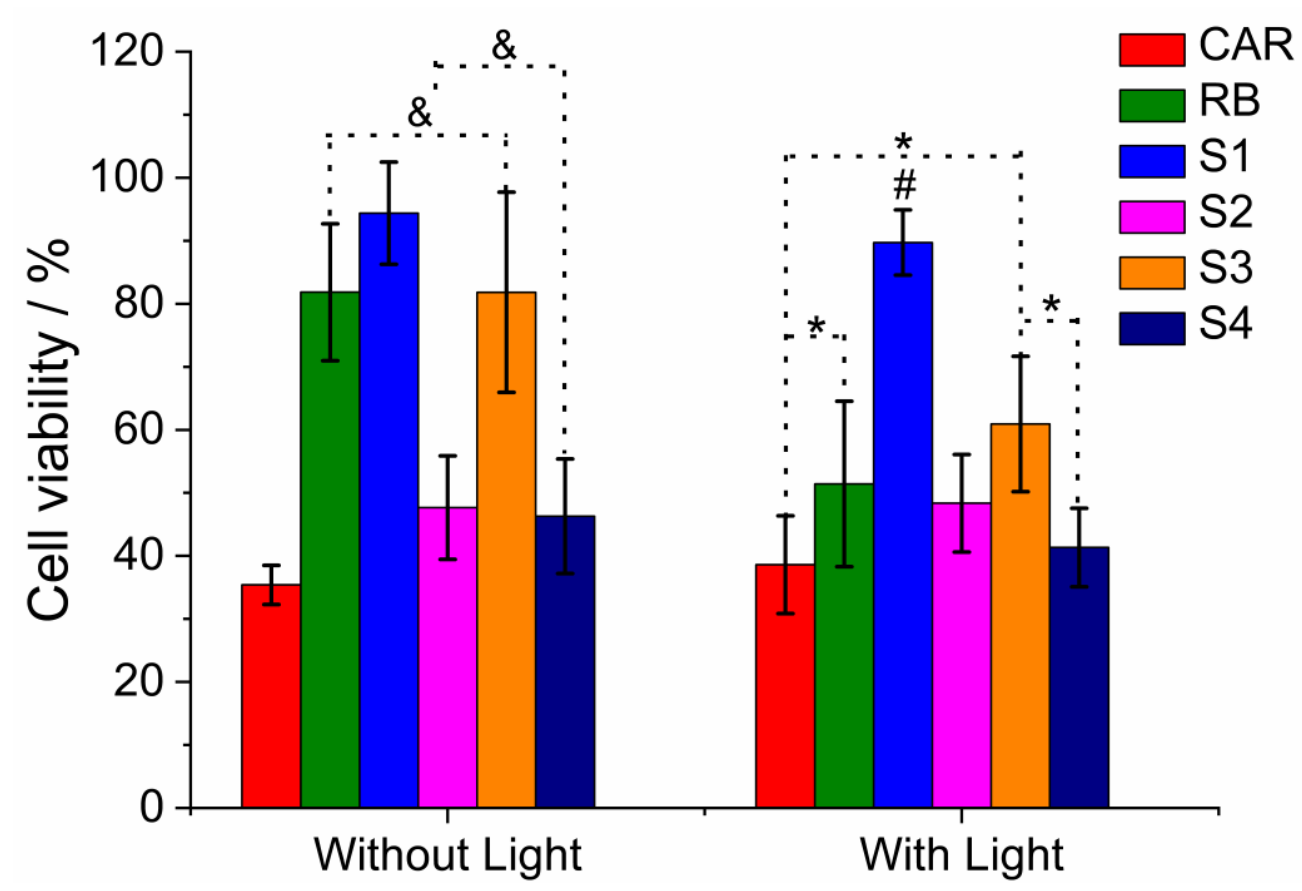

(a)

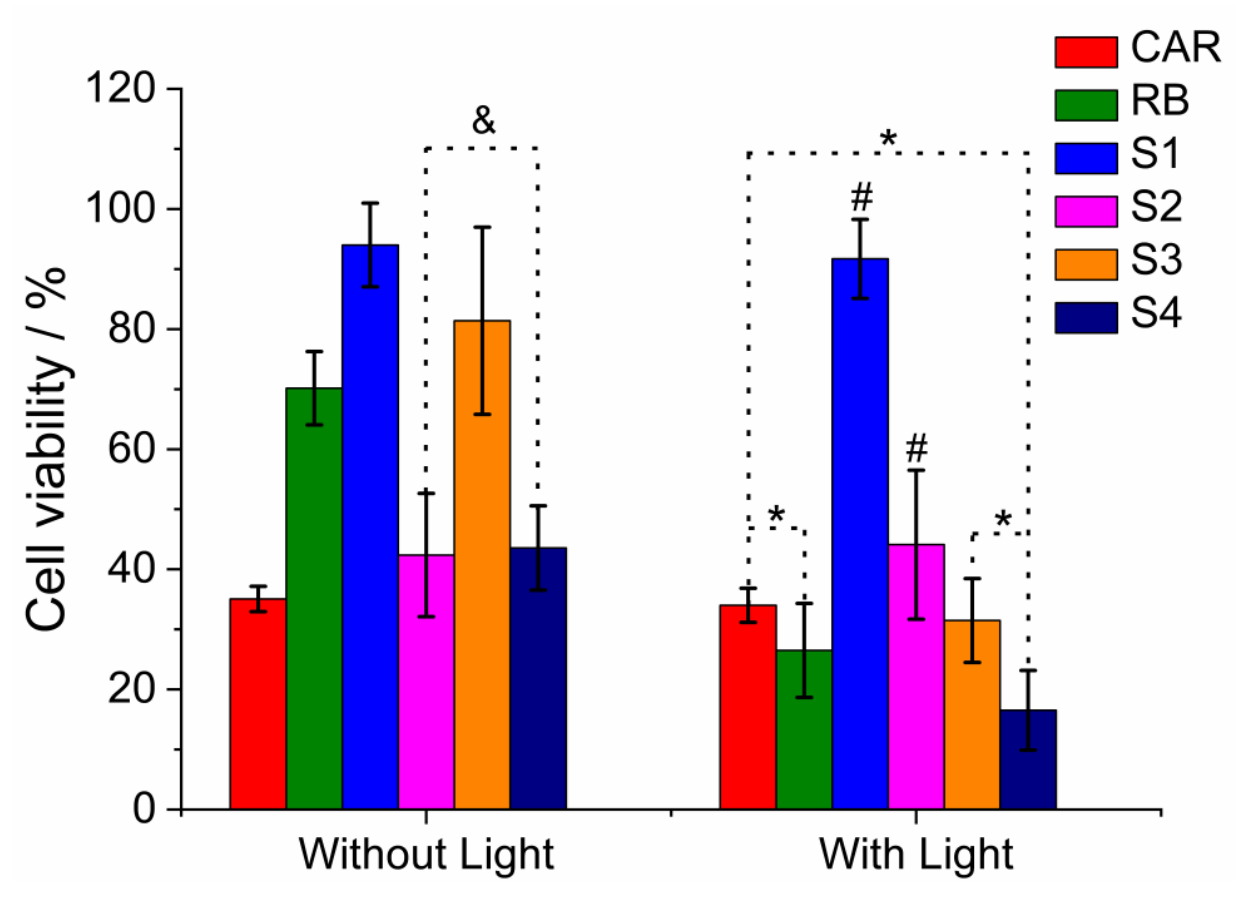

(b)

330 Figure 5. Cytotoxicity studies with (a) HDF and (b) Caco-2 cells. Data are shown from three independent experiments (with three replicate wells per experiment) as mean \pm S.D. Percentages are relative to an untreated cells control. CAR and RB denote solutions of the pure drugs. * indicates a significant difference between two groups $(p<0.05)$, \# a significant difference from all other groups $(p<0.05)$ and \& no significant difference between two groups $(p>0.05)$.

A selectivity index was calculated as the viability of HDF cells divided by the viability of Caco-2 cells, to give a measure of the ability of the formulations to specifically induce death in cancer cells over healthy cells (Table 
2). It can be seen that for $C A R, S 1$ and $S 2$ the selectivity indices are very close to 1 regardless of whether light was applied or not, indicating that the materials are equally toxic to both the cancerous and non-cancerous cells. The same is true for RB, S3 and S4 in the absence of light. However, when light is applied RB, S3 and S4 have selectivity indices ranging from $1.94-2.50$, showing enhanced toxicity to cancerous over non-cancerous cells. The highest value, 2.50 , was noted with the $\$ 4$ formulation.

Greater selectivity for cancerous over non-cancerous cells has been noted previously for electrosprayed poly(vinylpyrrolidone) particles [7], but those formulations were very rapid dissolving, and thus could not practicably be used for cancer treatment. The selectivity values observed in this work are of the same order of magnitude as other reports in the literature, for instance with graphene oxide based delivery systems [37] or Ru-based anticancer prodrugs [38]. Overall therefore, the coaxial fibers appear to have potential for combined photo-chemotherapy.

Table 2. Selectivity indices, calculated as viability of HDF cells / viability of Caco-2 cells.

\begin{tabular}{ccc}
\hline \multirow{2}{*}{ Sample } & \multicolumn{2}{c}{ Selectivity index } \\
\cline { 2 - 3 } & Without light & With light \\
\hline CAR & 1.01 & 1.03 \\
RB & 1.17 & 1.94 \\
S1 & 1.00 & 0.98 \\
S2 & 1.12 & 1.10 \\
S3 & 1.01 & 1.94 \\
S4 & 1.06 & 2.50 \\
\hline
\end{tabular}

\section{Mucoadhesion}

As the electrospun nanofibers loaded with both of the drugs showed promising effects in the cytotoxicity study, the $\mathbf{S 4}$ fibers were chosen as a representative sample with which to test the mucoadhesive properties of the formulations. The results are displayed in Figure 6. Compared with the negative control, blank HPMC nanofibers prepared by single-fluid spinning showed good mucoadhesion (1.89 $\pm 0.17 \mathrm{~mJ})$. This is in accordance with the literature, which reports HPMC to have good mucoadhesive properties [20,39].

To mimic the effects of passage through the gastric and intestinal fluids, the $\mathrm{S} 4$ nanofibers were first immersed in $\mathrm{HCl}$ and then transferred into PBS for various timeperiods before their adhesive properties were explored. The fibers immersed in $\mathrm{HCl}$ for $2 \mathrm{~h}$ showed low work of adhesion $(1.10 \pm 0.18 \mathrm{~mJ})$, because the Eudragit shell 
is insoluble in acidic media, and thus the HPMC core could not interact with the tissue. When the fibers were exposed to PBS, the shell dissolved and the HPMC core was exposed. After immersion in PBS for $1 \mathrm{~h}$, the work of adhesion increased to $1.50 \pm 0.37 \mathrm{~mJ}$. This was then largely maintained during longer incubation times, and after $4 \mathrm{~h}$ in PBS the work of adhesion was $1.33 \pm 0.28 \mathrm{~mJ}$. These results indicate the $\mathrm{S} 4$ fibers should adhere to the wall of the colon for at least $4 \mathrm{~h}$ after reaching the site of action. These values represent an improvement over a previous study using Eudragit S100 and a poly(ethylene oxide) core, where work of adhesion values of the order of $0.5-1 \mathrm{~mJ}$ were noted after the fibers had been immersed in $\mathrm{HCl}$ for $2 \mathrm{~h}$ and then exposed to a PBS buffer [24]. It is to be expected that the residence time of CAR and RB in the colon could be increased using the core/shell fibers because of the existence of mucoadhesive HPMC in the core, which is promising for targeting tumors into the colonic region.

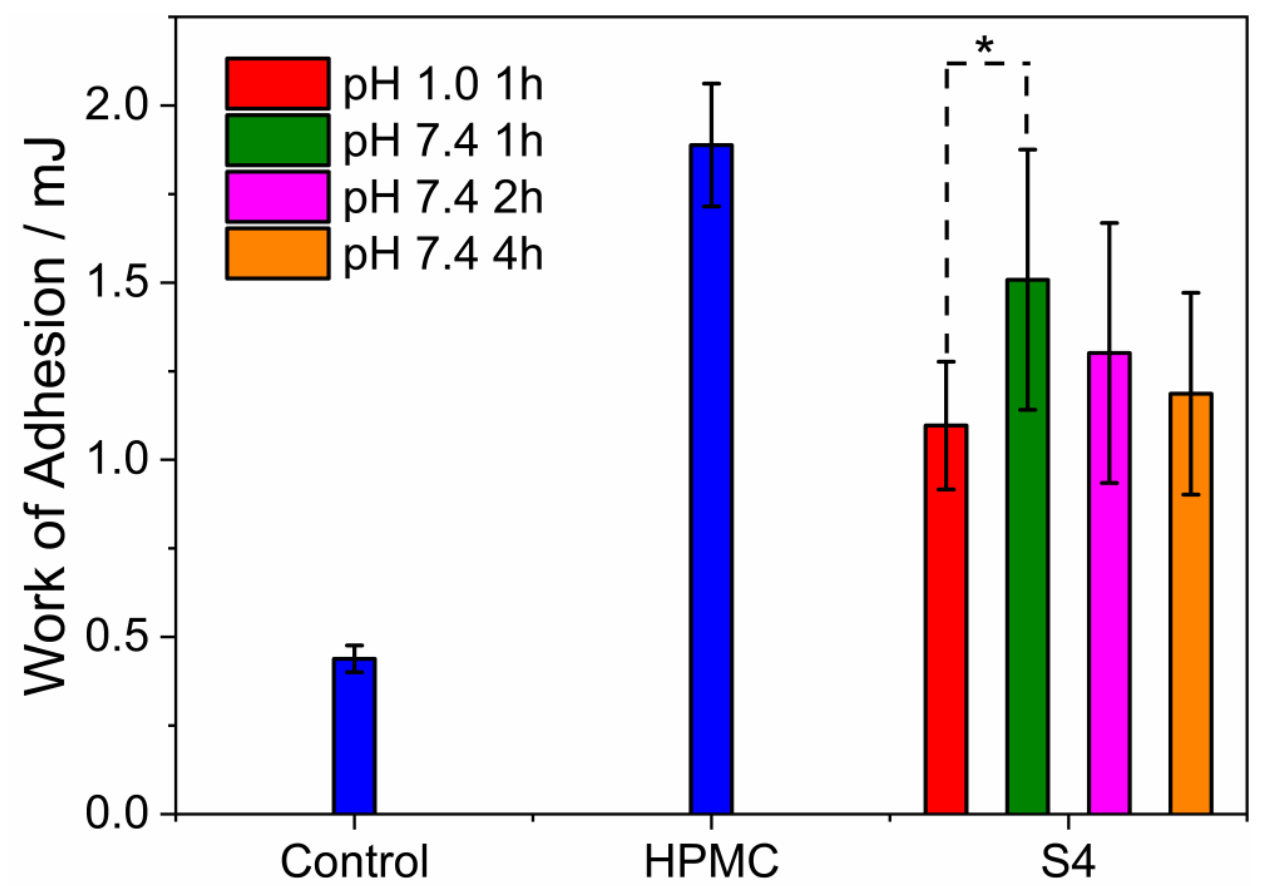

Figure 6. Mucoadhesion results. Data are shown from three independent experiments as mean \pm S.D. ${ }^{*}$ indicates a significant difference between two groups $(p<0.05)$.

\section{Conclusions}

Core/shell fibers loaded with the chemotherapeutic drug carmofur (CAR) and the photosensitizer rose bengal (RB) were successfully fabricated by coaxial electrospinning. The fibers consist of a Eudragit L100-55 shell and a hydroxypropyl methylcellulose core, with the latter loaded with the active ingredient(s). Scanning electron microscopy images revealed the fibers to be largely homogeneous and cylindrical, with smooth surfaces. 
Transmission electron microscopy confirmed all the fibers to have clear core-shell architectures. A physical form assessment was performed using X-ray diffraction and differential scanning calorimetry, and the fibers are shown to comprise amorphous solid dispersions. During in vitro drug release tests only $10-15 \%$ drug release was noted in acidic conditions, as a result of the Eudragit shell precluding drug release from the core. At $\mathrm{pH} 7.4$ the shell dissolves and sustained release of the incorporated active ingredients over $4-8 \mathrm{~h}$ is observed. Cell viability studies were undertaken using representative healthy (HDF) and cancerous (Caco-2) cell lines. While the CAR-loaded materials caused widespread cell death of both cell lines, with and without light exposure, the RB- and RB/CAR dual-loaded fibers provided a greater degree of selectivity for cancerous cells. Finally, mucoadhesion tests were performed, and revealed that the fibers can adhere to the wall of the colon for at least $4 \mathrm{~h}$. Therefore, the CAR and RB-loaded fibers prepared in this work could comprise a potent candidate therapy method to treat colon cancer via oral administration.

\section{Acknowledgements}

395 The authors gratefully thank: the China Scholarship Council for funding (award to HL to study at University College London); CONACyT for the provision of a PhD studentship to BSV; and, the Engineering and Physical Sciences Research Council (EPSRC) for PhD funding to RPT through the Centre for Doctoral Training in Advanced Therapeutics \& Nanomedicines (EP/L01646X/1). We are also grateful for Dr Andrew Weston for assistance with electron microscopy experiments.

\section{References}

[1] R.L. Siegel, K.D. Miller, S.A. Fedewa, D.J. Ahnen, R.G.S. Meester, A. Barzi, A. Jemal, Colorectal cancer statistics, 2017, CA: A Cancer Journal for Clinicians, 67 (2017) 177-193.

[2] T. André, C. Boni, L. Mounedji-Boudiaf, M. Navarro, J. Tabernero, T. Hickish, C. Topham, M. Zaninelli, P. Clingan, J. Bridgewater, I. Tabah-Fisch, A.d. Gramont, Oxaliplatin, fluorouracil, and leucovorin as adjuvant treatment for colon cancer, New England Journal of Medicine, 350 (2004) 2343-2351.

[3] T. André, C. Boni, M. Navarro, J. Tabernero, T. Hickish, C. Topham, A. Bonetti, P. Clingan, J. Bridgewater, F. Rivera, A.d. Gramont, Improved overall survival with oxaliplatin, fluorouracil, and leucovorin as adjuvant treatment in sage II or III colon cancer in the MOSAIC yrial, Journal of Clinical Oncology, 27 (2009) 3109-3116.

410 [4] A. de Gramont, E. Van Cutsem, H.-J. Schmoll, J. Tabernero, S. Clarke, M.J. Moore, D. Cunningham, T.H. Cartwright, J.R. Hecht, F. Rivera, S.-A. Im, G. Bodoky, R. Salazar, F. Maindrault-Goebel, E. Shacham-Shmueli, E. Bajetta, M. Makrutzki, A. Shang, T. André, P.M. Hoff, Bevacizumab plus oxaliplatin-based chemotherapy as adjuvant treatment for colon cancer (AVANT): a phase 3 randomised controlled trial, The Lancet Oncology, 13 (2012) 1225-1233.

[5] D.E.J.G.J. Dolmans, D. Fukumura, R.K. Jain, Photodynamic therapy for cancer, Nature Reviews Cancer, 3 (2003) 380 387. 
[6] G. Pasparakis, T. Manouras, M. Vamvakaki, P. Argitis, Harnessing photochemical internalization with dual degradable nanoparticles for combinatorial photo-chemotherapy, Nature Communications, 5 (2014) 3623.

[7] B. Sanchez-Vazquez, A.J.R. Amaral, D.G. Yu, G. Pasparakis, G.R. Williams, Electrosprayed Janus particles for combined photo-chemotherapy, AAPS PharmSciTech, 18 (2017) 1460-1468.

420 [8] E.-K. Lim, T. Kim, S. Paik, S. Haam, Y.-M. Huh, K. Lee, Nanomaterials for theranostics: Recent advances and future challenges, Chemical Reviews, 115 (2015) 327-394.

[9] N. Bhardwaj, S.C. Kundu, Electrospinning: A fascinating fiber fabrication technique, Biotechnology Advances, 28 (2010) 325-347.

[10] G.R. Williams, B.T. Raimi-Abraham, C.J. Luo, Nanofibres in drug delivery, UCL Press, London, 2018.

$425[11]$ X. Hu, S. Liu, G. Zhou, Y. Huang, Z. Xie, X. Jing, Electrospinning of polymeric nanofibers for drug delivery applications, Journal of Controlled Release, 185 (2014) 12-21.

[12] A.C. Mendes, C. Gorzelanny, N. Halter, S.W. Schneider, I.S. Chronakis, Hybrid electrospun chitosan-phospholipids nanofibers for transdermal drug delivery, International Journal of Pharmaceutics, 510 (2016) 48-56.

[13] X. Wang, D.G. Yu, X.Y. Li, S.W. Bligh, G.R. Williams, Electrospun medicated shellac nanofibers for colon-targeted drug delivery, Internationa Journal of Pharmaceutics, 490 (2015) 384-390.

[14] E.A. Krogstad, K.A. Woodrow, Manufacturing scale-up of electrospun poly(vinyl alcohol) fibers containing tenofovir for vaginal drug delivery, International Journal of Pharmaceutics, 475 (2014) 282-291.

[15] S. Seif, L. Franzen, M. Windbergs, Overcoming drug crystallization in electrospun fibers - Elucidating key parameters and developing strategies for drug delivery, International Journal of Pharmaceutics, 478 (2015) 390-397.

435 [16] Z. Sultanova, G. Kaleli, G. Kabay, M. Mutlu, Controlled release of a hydrophilic drug from coaxially electrospun polycaprolactone nanofibers, International Journal of Pharmaceutics, 505 (2016) 133-138.

[17] Y. Chen, S. Liu, Z. Hou, P. Ma, D. Yang, C. Li, J. Lin, Multifunctional electrospinning composite fibers for orthotopic cancer treatment in vivo, Nano Research, 8 (2015) 1917-1931.

[18] J.A. Kaplan, R. Liu, J.D. Freedman, R. Padera, J. Schwartz, Y.L. Colson, M.W. Grinstaff, Prevention of lung cancer recurrence using cisplatin-loaded superhydrophobic nanofiber meshes, Biomaterials, 76 (2016) 273-281.

[19] L. Poláková, J. Širc, R. Hobzová, A.-I. Cocârță, E. Heřmánková, Electrospun nanofibers for local anticancer therapy: Review of in vivo activity, International Journal of Pharmaceutics, 558 (2019) 268-283.

[20] E. Karavas, E. Georgarakis, D. Bikiaris, Application of PVP/HPMC miscible blends with enhanced mucoadhesive properties for adjusting drug release in predictable pulsatile chronotherapeutics, European Journal of Pharmaceutics and Biopharmaceutics, 64 (2006) 115-126.

[21] G. Ponchel, Formulation of oral mucosal drug delivery systems for the systemic delivery of bioactive materials, Advanced Drug Delivery Reviews, 13 (1994) 75-87.

[22] G.P. Andrews, T.P. Laverty, D.S. Jones, Mucoadhesive polymeric platforms for controlled drug delivery, European Journal of Pharmaceutics and Biopharmaceutics, 71 (2009) 505-518.

450 [23] C. Dott, C. Tyagi, L.K. Tomar, Y.E. Choonara, P. Kumar, L.C. du Toit, V. Pillay, A mucoadhesive electrospun nanofibrous natrix for rapid oramucosal drug delivery, Journal of Nanomaterials, 2013 (2013) 1-19.

[24] M. Jin, D.G. Yu, X. Wang, C.F. Geraldes, G.R. Williams, S.W. Bligh, Electrospun contrast-agent-loaded fibers for colontargeted MRI, Advanced Healthcare Materials, 5 (2016) 977-985.

[25] F.J.O. Varum, F. Veiga, J.S. Sousa, A.W. Basit, Mucoadhesive platforms for targeted delivery to the colon, International 455 Journal of Pharmaceutics, 420 (2011) 11-19.

[26] V.U. Godakanda, H. Li, L. Alquezar, L. Zhao, L.M. Zhu, R. de Silva, K.M.N. de Silva, G.R. Williams, Tunable drug release from blend poly(vinyl pyrrolidone)-ethyl cellulose nanofibers, Interational Journal of Pharmaceutics, 562 (2019) $172-179$. [27] U.E. Illangakoon, H. Gill, G.C. Shearman, M. Parhizkar, S. Mahalingam, N.P. Chatterton, G.R. Williams, Fast dissolving paracetamol/caffeine nanofibers prepared by electrospinning, International Journal of Pharmaceutics, 477 (2014) 369 379. 
[28] H. Li, M. Wang, G.R. Williams, J. Wu, X. Sun, Y. Lv, L.-M. Zhu, Electrospun gelatin nanofibers loaded with vitamins A and $\mathrm{E}$ as antibacterial wound dressing materials, RSC Advances, 6 (2016) 50267-50277.

[29] J.M. Deitzel, J. Kleinmeyer, D. Harris, N.C. Beck Tan, The effect of processing variables on the morphology of electrospun nanofibers and textiles, Polymer, 42 (2001) 261-272.

[30] Y.-T. Jia, J. Gong, X.-H. Gu, H.-Y. Kim, J. Dong, X.-Y. Shen, Fabrication and characterization of poly (vinyl alcohol)/chitosan blend nanofibers produced by electrospinning method, Carbohydrate Polymers, 67 (2007) $403-409$.

[31] H. McPhillips, D.Q.M. Craig, P.G. Royall, V.L. Hill, Characterisation of the glass transition of HPMC using modulated temperature differential scanning calorimetry, International Journal of Pharmaceutics, 180 (1999) 83-90.

[32] S. Kuzuhara, N. Ohkoshi, K. Kanemaru, H. Hashimoto, T. Nakanishi, Y. Toyokura, Subacute leucoencephalopathy 470 induced by carmofur, a 5-fluorouracil derivative, Journal of Neurology, 234 (1987) 365-370.

[33] K.T. Douglas, The thymidylate synthesis cycle and anticancer drugs, Medicinal Reseach Reviews, 7 (1987) $441-475$.

[34] B.C. Wilson, Photodynamic therapy for cancer: Principles, Can. J. Gastroenterol., 16 (2002) 393-396.

[35] G. Canti, A. Nicolin, R. Cubeddu, P. Taroni, G. Bandieramonte, G. Valentini, Antitumor efficacy of the combination of photodynamic therapy and chemotherapy in murine tumors, Cancer Letters, 125 (1998) 39-44.

475 [36] L. Zhou, L. Zhou, S. Wei, X. Ge, J. Zhou, H. Jiang, F. Li, J. Shen, Combination of chemotherapy and photodynamic therapy using graphene oxide as drug delivery system, Journal of Photochemistry and Photobiology B: Biology, 135 (2014) 7-16.

[37] A. Sahu, W.I. Choi, J.H. Lee, G. Tae, Graphene oxide mediated delivery of methylene blue for combined photodynamic and photothermal therapy, Biomaterials, 34 (2013) 6239-6248.

480 [38] F.R. Qu, S. Park, K. Martinez, J.L. Gray, F.S. Thowfeik, J.A. Lundeen, A.E. Kuhn, D.J. Charboneau, D.L. Gerlach, M.M. Lockart, J.A. Law, K.L. Jernigan, N. Chambers, M. Zeller, N.A. Piro, W.S. Kassel, R.H. Schmehl, J.J. Paul, E.J. Merino, Y. Kim, E.T. Papish, Ruthenium xomplexes are $\mathrm{pH}$-activated metallo prodrugs (pHAMPs) with light-triggered selective toxicity toward cancer cells, Inorganic Chemistry, 56 (2017) 7519-7532.

[39] B.K. Jadhav, K.R. Khandelwal, A.R. Ketkar, S.S. Pisal, Formulation and evaluation of mucoadhesive tablets containing eugenol for the treatment of periodontal diseases, Drug Development and Industrial Pharmacy, 30 (2004) $195-203$. 